\title{
Tri-Resistive Switching Behavior of Hydrogen Induced Resistance Random Access Memory
}

\author{
Tian-Jian Chu, Tsung-Ming Tsai, Ting-Chang Chang, Senior Member, IEEE, Kuan-Chang Chang, Rui Zhang, \\ Kai-Huang Chen, Jung-Hui Chen, Tai-Fa Young, Jen-Wei Huang, Jen-Chung Lou, Min-Chen Chen, \\ Syuan-Yong Huang, Hsin-Lu Chen, Yong-En Syu, Dinghua Bao, and Simon M. Sze, Life Fellow, IEEE
}

\begin{abstract}
In this letter, the special role of hydrogen ions in hafnium doped silicon oxide resistive random access memory (RRAM) is presented. In addition to the more typical oxygen iondominated resistive switching, hydrogen ions were also observed to trigger a resistance transformation phenomenon, producing a tri-resistive device. Unlike a normal RRAM device, a hydrogen plasma-treated device is operated with a reversed voltage polarity, and the direction of hydrogen ion migration results in the chemical bonds breaking and repairing. By changing the voltage polarity and stop voltage, this tri-resistive behavior can be achieved. This particular hydrogen-induced switching behavior suggests a different RRAM switching mechanism and is finally explained by our model.
\end{abstract}

Index Terms-RRAM, hydrogen, resistive switching, triresistive states.

\section{INTRODUCTION}

$\mathbf{T}$ O OVERCOME the technical and physical limitation challenges of nonvolatile floating memory, next generation nonvolatile memories (NVM) have been researched exten-

Manuscript received November 19, 2013; revised December 10, 2013; accepted December 12, 2013. Date of publication January 2, 2014; date of current version January 23, 2014. The work was supported in part by the National Science Council of China under Contracts NSC-101-2120-M-110001, and NSC 101-2221-E-110-044-MY3, in part by the National Science Council Core Facilities Laboratory for Nano-Science and Nano-Technology in Kaohsiung-Pingtung area, and in part by the NSYSU Center for NanoScience and Nano-Technology. The review of this letter was arranged by Editor C. V. Mouli.

T.-J. Chu, T.-M. Tsai, K.-C. Chang, and S.-Y. Huang are with the Department of Materials and Optoelectronic Science, National Sun Yat-Sen University, Kaohsiung 804, Taiwan.

T.-C. Chang, M.-C. Chen, and Y.-E. Syu are with the Department of Physics, National Sun Yat-Sen University, Kaohsiung 804, Taiwan, and also with the Advanced Optoelectronics Technology Center, National Cheng Kung University, Tainan 701, Taiwan (e-mail: tcchang@mail.phys.nsysu.edu.tw).

R. Zhang and J.-C. Lou are with the School of Software and Microelectronics, Peking University, Beijing 100871, China.

K.-H. Chen is with the Department of Electronics Engineering and Computer Science, Tung-Fang Design Institute, Kaohsiung 829, Taiwan.

J.-H. Chen is with the Department of Chemistry, National Kaohsiung Normal University, Kaohsiung 804, Taiwan.

T.-F. Young and H.-L. Chen are with the Department of Mechanical and Electro-Mechanical Engineering, National Sun Yat-Sen University, Kaohsiung 804, Taiwan.

J.-W. Huang is with the Department of Physics, R.O.C. Military Academy, Kaohsiung 83055, Taiwan.

D. Bao is with the State Key Laboratory of Optoelectronic Materials and Technologies, School of Physics and Engineering, Sun Yat-Sen University, Guangzhou 510275, China.

S. M. Sze is with the Department of Electronics Engineering, National Chiao Tung University, Hsinchu 300, Taiwan.

Color versions of one or more of the figures in this letter are available online at http://ieeexplore.ieee.org.

Digital Object Identifier 10.1109/LED.2013.2295378 sively [1]-[4]. The resistance random access memory (RRAM) device has great potential as next generation nonvolatile memory device owing to its advantages of low operating power, fast operation speed and high density integration [5]-[8].

Metal-doped $\mathrm{SiO}_{2}$-based RRAM devices have shown bipolar switching behavior which can be "set" [switching from the high-resistance state (HRS) to the low-resistance state (LRS)] when applied with positive bias and "reset" by applying negative bias. Moreover, outstanding performance has been reported in $\mathrm{SiO}_{2}$-based RRAM devices, including over $10^{6}$ cycles endurance and excellent retention under $85^{\circ} \mathrm{C}$ [9].

Metal-doped silicon oxide $\left(\mathrm{M}: \mathrm{SiO}_{\mathrm{x}}\right)$ material used as a resistive switching layer has been studied in our previous work [10]-[13]. In this letter, sandwiched $\mathrm{Pt} / \mathrm{Hf}_{\mathrm{SiO}} \mathrm{Si}_{\mathrm{x}} / \mathrm{TiN}$ devices were fabricated to investigate the effect of hydrogen ions in the resistive switching process of $\mathrm{Hf}_{\mathrm{S}} \mathrm{SiO}_{\mathrm{x}}$ devices. Types of chemical bonds were analyzed by a Fourier transform infrared spectroscope (FTIR), and current conduction fitting was applied to evaluate the switching properties of the $\mathrm{Hf}: \mathrm{SiO}_{\mathrm{x}}$ devices [14].

\section{EXPERIMENTAL SETUP}

The fabrication process of RRAM device was conducted over a polished $\mathrm{p}$-Si wafer of nominal resistively $\sim 1.0 \Omega$-cm. To overcome the existence of native-oxide on p-type silicon wafer, the particles and metal ions on p-type silicon wafer were removed during RCA clean process. In order to remove the defects of metal target and obtain stable plasma during sputtering deposition time, pre-sputtering time of the target was maintained for 10 minutes under argon ambient.

The experimental specimens were prepared as follows: a $\mathrm{Hf}: \mathrm{SiO}_{\mathrm{x}}$ thin film (about $10 \mathrm{~nm}$ ) was deposited on the TiN/Ti/SiO $/ 2 / \mathrm{Si}$ substrate by co-sputtering with pure $\mathrm{SiO}_{2}$ and $\mathrm{Hf}$ targets. On contrast, one $\mathrm{Hf}: \mathrm{SiO}_{\mathrm{x}}$ film sample was subsequently treated by hydrogen plasma. Ultimately, the Pt top electrode with a thickness of $200 \mathrm{~nm}$ was deposited on both (with and without hydrogen plasma treatment) $\mathrm{Hf}: \mathrm{SiO}_{\mathrm{x}}$ devices to form $\mathrm{Pt} / \mathrm{Hf}: \mathrm{SiO}_{\mathrm{x}} / \mathrm{TiN}$ sandwich structure. The electrical measurements of devices were performed using an Agilent B1500A semiconductor parameter analyzer.

\section{RESUlTS AND DISCUSSION}

By applying different stop voltages, the hydrogen plasmatreated RRAM devices exhibited special tri-resistive states. First, a linear voltage sweep from $0 \mathrm{~V}$ to $0.6 \mathrm{~V}$ was applied to 


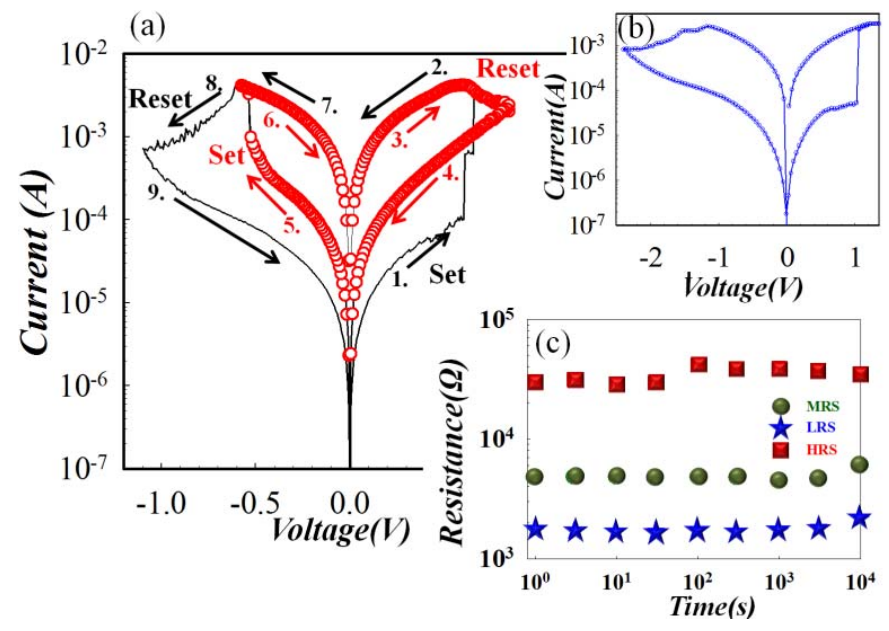

Fig. 1. (a) I-V resistive switching characteristics of hydrogen plasma-treated $\mathrm{Pt} / \mathrm{Hf}: \mathrm{SiO}_{\mathrm{x}} / \mathrm{TiN}$ device for both counterclockwise/oxygen-dominated (black) and clockwise/hydrogen-dominated (red) switching, dependent on voltage polarity. (b) The typical $\mathrm{I}-\mathrm{V}$ curve properties of an untreated $\mathrm{Hf}: \mathrm{SiO}_{\mathrm{x}}$ device. (c) The retention characteristics and the resistive ratio within LRS/MRS/HRS remain at about one order of magnitude even after $10^{4}$ seconds at $85{ }^{\circ} \mathrm{C}$.

the device in order to switch RRAM from HRS to LRS. After that, a larger sweeping range from $0 \mathrm{~V}$ to $0.9 \mathrm{~V}$ was applied to switch the device from LRS to the middle resistance state (MRS). Then MRS can be changed to LRS with a negative sweep from $0 \mathrm{~V}$ to $-0.5 \mathrm{~V}$. Finally, we can achieve HRS again by applying another negative voltage sweep with a range of $0 \mathrm{~V} \sim-1.1 \mathrm{~V}$. Thus tri-resistive switching behavior was achieved by changing the operating voltage polarity and stop voltage. The whole operating procedures are demonstrated in Fig. 1(a).

For comparison, the typical switching behavior of $\mathrm{Hf}: \mathrm{SiO}_{\mathrm{x}}$ (without hydrogen plasma treatment) devices which have only two resistive states is shown in Fig. 1(b). The device was switched to LRS by applying a linear voltage sweep to $1.3 \mathrm{~V}$ and then was back to HRS by applying negative voltage with a range from $0 \mathrm{~V}$ to $-2.4 \mathrm{~V}$.

To further investigate the reliability of the devices, retention properties were measured. Three resistance states were extracted with a reading voltage of 0.1 V. Fig. 1(c) shows that the retention characteristics and the resistive ratio within LRS/MRS/HRS remained at about one order of magnitude after $10^{4}$ seconds at $85^{\circ} \mathrm{C}$.

To analyze the influence of hydrogen plasma on resistance switching characteristics, FTIR spectroscopy was used. Complying with the Beer's law, the absorption coefficient and the thickness of the film did not change after hydrogen plasma treatment while the intensity of signal was proportional to the concentration of the corresponding bonds. By comparing the FTIR spectra of the $\mathrm{Hf}: \mathrm{SiO}_{\mathrm{x}}$ films (with and without treatment), we found that the intensity of $\mathrm{Hf}-\mathrm{O}$ stretch mode bonding at $595 \mathrm{~cm}^{-1}$ decreases after the treatment. In addition, the absorption of the bending, symmetric and asymmetric stretch modes of $\mathrm{Si}-\mathrm{O}-\mathrm{Si}$ bonds at $500 \mathrm{~cm}^{-1}, 1050 \mathrm{~cm}^{-1}$ and $1100 \mathrm{~cm}^{-1}$ also decreased after the hydrogen plasma treatment [15]. On the contrary, the intensity of stretch mode of $\mathrm{O}-\mathrm{H}$ bonding between $3400 \mathrm{~cm}^{-1}$ and $3600 \mathrm{~cm}^{-1}$ increased,

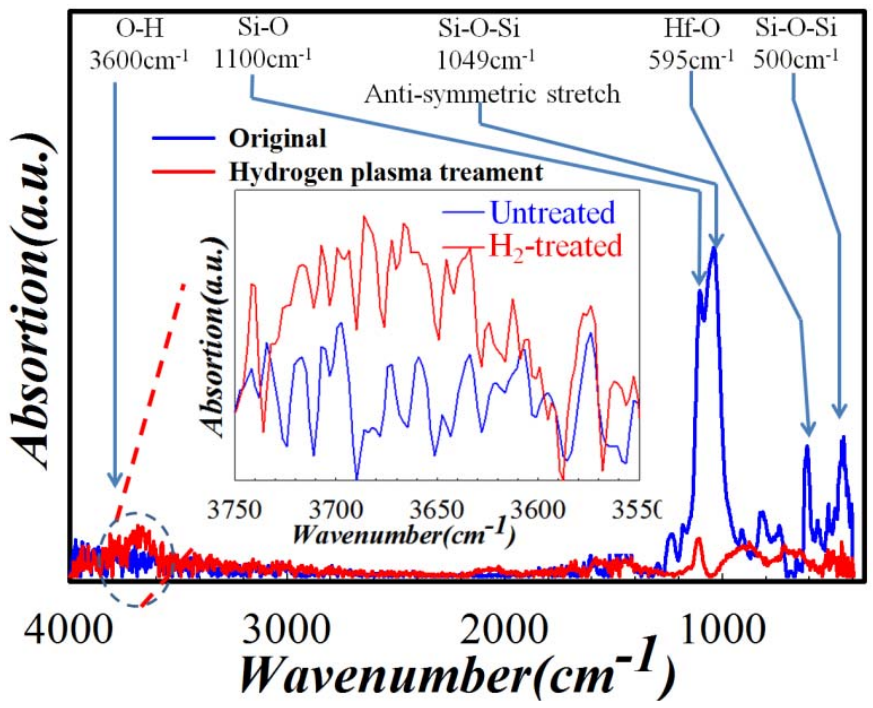

Fig. 2. Comparison of FTIR spectra of $\mathrm{Hf}_{\mathrm{SiO}} \mathrm{x}$ film before and after hydrogen plasma treatment. The anti-symmetric stretch mode intensity of $\mathrm{Si}-\mathrm{O}-\mathrm{Si}$ and $\mathrm{Si}-\mathrm{O}$ bonds decreased. The enlarged view around wavenumber $3600 \mathrm{~cm}^{-1}$ indicated the increase for stretch mode intensity of $\mathrm{O}-\mathrm{H}$ bond after hydrogen plasma treatment.

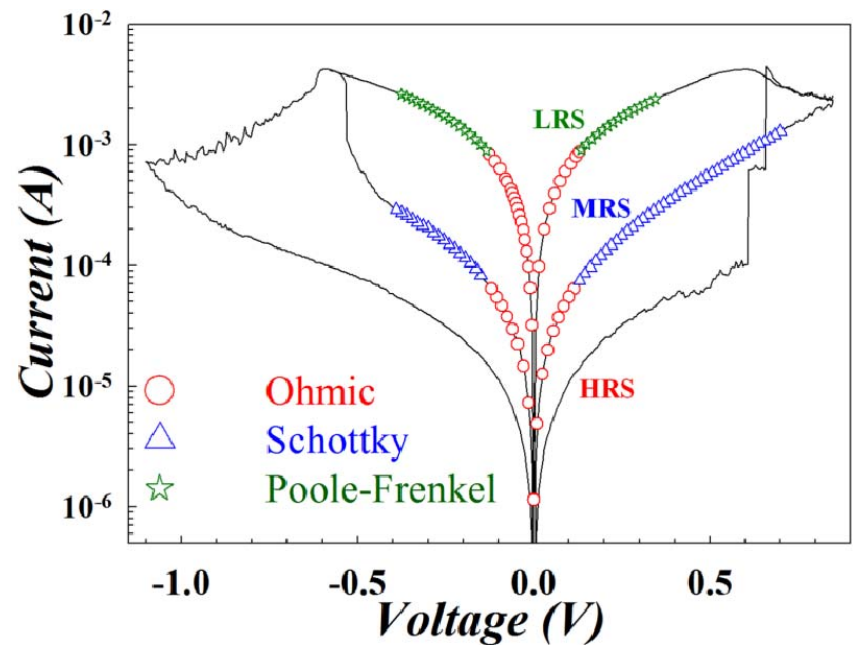

Fig. 3. Current conduction fitting of LRS and MRS.

as shown in the inset of Fig. 2. From Fig. 2, we can conclude that the level of oxidation in $\mathrm{Hf}_{\mathrm{SiO}} \mathrm{x}$ film decreased after hydrogen plasma treatment.

The current conduction mechanisms of MRS and LRS were analyzed in order to further discuss the resistance switching properties in the hydrogen plasma-treated RRAM. The current conduction fitting results are shown in Fig. 3.

The I-V fitting results show that LRS $(0.13-0.35 \mathrm{~V}$, and $-0.14-0.4 \mathrm{~V}$.) is dominated by the Poole-Frenkel emission mechanism according to the relationship of Poole-Frenkel conduction, I $\propto \mathrm{V} \exp \left[(\mathrm{q} / \mathrm{KT})\left(2 \mathrm{a} \sqrt{\mathrm{V}}-\varphi_{\mathrm{Bt}}\right)\right]$, where a is $\sqrt{q} / 4 \pi \mathrm{d} \varepsilon_{\mathrm{i}}, \quad \varphi_{\mathrm{Bt}}$ is the trap barrier height, and $\mathrm{d}$ is the insulator thickness. The current conduction mechanism in MRS $(0.13-0.7 \mathrm{~V}$, and $-0.13-0.4 \mathrm{~V}$.) is dominated by Schottky conduction, according to the relationship $\mathrm{J}=\mathrm{A}^{*} \mathrm{~T} 2 \exp \left[-\mathrm{q}\left(\varphi \mathrm{B} \sqrt{q E / 4 \pi \varepsilon_{\mathrm{i}}}\right) / \mathrm{kT} \cdot \mathrm{A}^{*}\right.$ is effective 


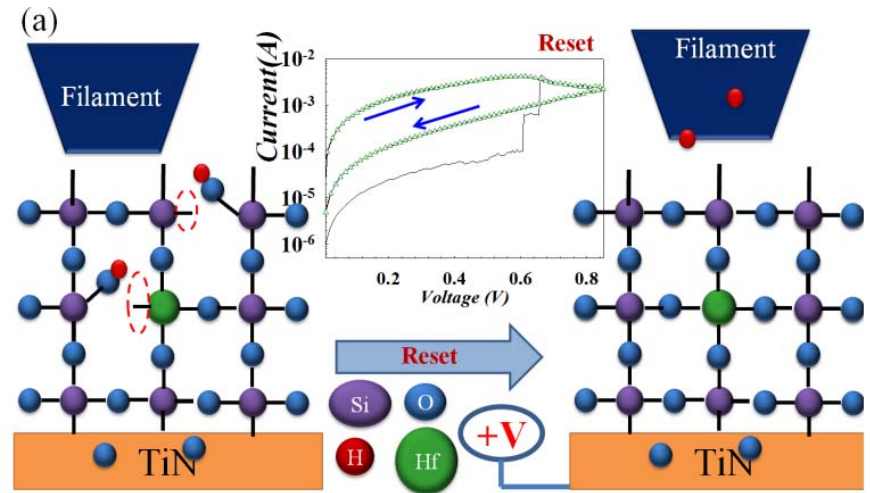

(b)

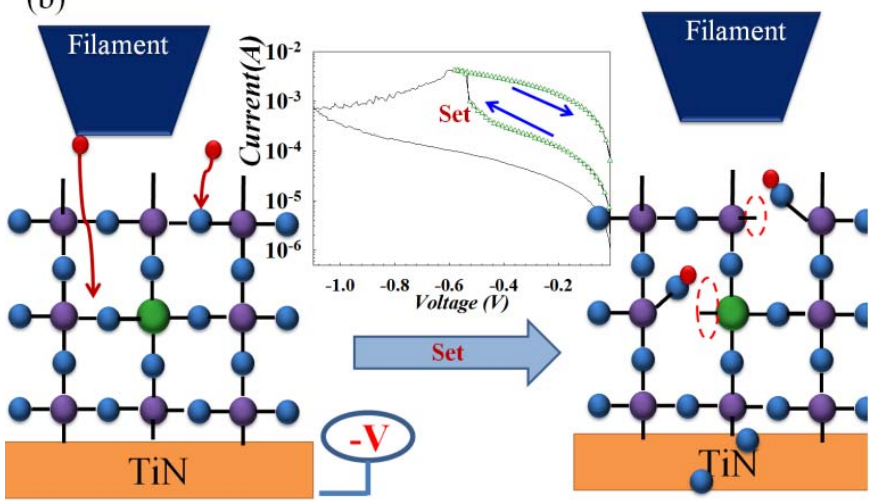

Fig. 4. Schematic diagrams of (a) dehydrogen and rebonding effect and (b) bond breaking and hydrogen rebonding effect in hydrogen-induced RRAM.

Richardson constant, $\mathrm{q} \varphi_{\mathrm{B}}=$ barrier height and $\varepsilon_{\mathrm{i}}=\varepsilon_{0} \varepsilon_{\mathrm{r}}$, in which $\varepsilon_{\mathrm{r}}$ is the relative dielectric constant.

Combining the oxidation-reduction reaction analysis discussed above with the conduction current fitting results shown in Fig. 3, we propose a model to explain the electrical characteristics of the hydrogen-induced resistive switching behaviors, as shown in Fig. 4(a) and (b). After the forming process, film defects are generated owing to the generation of dangling bonds, leading to Poole-Frenkel conduction in LRS. With an increase in positive voltage, the hydrogen ions are released from $\mathrm{Si}-\mathrm{O}-\mathrm{H}$ bonds, after which the defects in the film will be repaired by cross linking due to the bonding of $\mathrm{Si}-\mathrm{O}-\mathrm{Si}$ and Si-O-Hf. Thus the reaction will lead to the transformation of MRS current conduction mechanism to Schottky conduction. These hydrogen ions are attracted to the bottom electrode when negative bias is applied, resulting in $\mathrm{Si}-\mathrm{O}-\mathrm{Si}$ and Si-O-Hf bonds breaking because of the hydrogen ions migration. Once again the density of defects increases owing to the breaking bonds, from which we can observe Poole-Frenkel conduction in LRS.

\section{CONCLUSION}

In conclusion, by applying hydrogen plasma treatment the hydrogen-induced RRAM can be operated with an opposite voltage polarity compared with common RRAM devices.
Based on the material and current conduction mechanism analysis, a model has been proposed to explain the special electrical characteristics. Extraordinary tri-resistive switching phenomenon can be observed in our device due to oxygen and hydrogen alternating in their domination of the resistive switching mechanism; a hydrogen ion-dominated mechanism is embedded in the typical oxygen-dominated switching mechanism. This thorough analysis of hydrogen-induced resistance switching will assist us better understanding the RRAM switching mechanism.

\section{REFERENCES}

[1] P. Pavan, R. Bez, P. Olivo, et al., "Flash memory cells-An overview," Proc. IEEE, vol. 85, no. 8, pp. 1248-1271, Aug. 1997.

[2] T. C. Chang, F. Y. Jian, S. C. Chen, et al., "Developments in nanocrystal memory," Mater. Today, vol. 14, no. 12, pp. 608-615, Dec. 2011.

[3] D. D. Jiang, M. H. Zhang, Z. L. Huo, et al., "A study of cycling induced degradation mechanisms in Si nanocrystal memory devices," Nanotechnology, vol. 22, no. 25, pp. 254009-1-254009-5, Jun. 2011

[4] S. Q. Yang, Q. Wang, M. H. Zhang, et al., "Titanium-tungsten nanocrystals embedded in $\mathrm{SiO}_{2} / \mathrm{Al}_{2} \mathrm{O}_{3}$ gate dielectric stack for low-voltage operation in non-volatile memory," Nanotechnology, vol. 21, no. 24, pp. 245201-245205, Jun. 2010.

[5] K. C. Chang, R. Zhang, T. C. Chang, et al., "Origin of hopping conduction in graphene-oxide-doped silicon oxide resistance random access memory devices," IEEE Electron Device Lett., vol. 34, no. 5, pp. 677-679, May 2013.

[6] M. C. Chen, T. C. Chang, C. T. Tsai, et al., "Influence of electrode material on the resistive memory switching property of indium gallium zinc oxide thin films," Appl. Phys. Lett., vol. 96, no. 26, pp. 262110-1-262110-3, Jun. 2010.

[7] Q. Liu, S. B. Long, H. B. Lv, et al., "Controllable growth of nanoscale conductive filaments in solid-electrolyte-based ReRAM by using a metal nanocrystal covered bottom electrode," ACS Nano, vol. 4, no. 10, pp. 6162-6168, 2010.

[8] T. J. Chu, T. C. Chang, T.-M. Tsai, et al., "Charge quantity influence on resistance switching characteristic during forming process," IEEE Electron Device Lett., vol. 34, no. 4, pp. 502-504, Apr. 2013.

[9] Y. T. Chen, B. Fowler, Y. Wang et al., "Tristate operation in resistive switching of $\mathrm{SiO}_{2}$ thin films," IEEE Electron Device Lett., vol. 33 , no. 12, pp. 1702-1704, Dec. 2012.

[10] T. M. Tsai, K. C. Chang, T. C. Chang, et al., "Bipolar resistive RAM characteristics induced by nickel incorporated into silicon oxide dielectrics for IC applications," IEEE Electron Device Lett., vol. 33, no. 12, pp. 1696-1698, Dec. 2012.

[11] T. M. Tsai, K. C. Chang, T. C. Chang, et al., "Origin of hopping conduction in Sn-doped silicon oxide RRAM with supercritical $\mathrm{CO}_{2}$ fluid treatment," IEEE Electron Device Lett., vol. 33, no. 12, pp. 1693-1695, Dec. 2012.

[12] K. C. Chang, T. M. Tsai, T. C. Chang, et al., "The effect of silicon oxide based RRAM with tin doping," Electrochem. Solid-State Lett., vol. 15, no. 3, pp. H65-H68, 2012.

[13] Y. E. Syu, T. C. Chang, J. H. Lou, et al., "Atomic-level quantized reaction of HfOx memristor," Appl. Phys. Lett., vol. 102, no. 7, pp. 172903-1-172903-4, Apr. 2013.

[14] Y. E. Syu, T. C. Chang, T. M. Tsai, et al., "Redox reaction switching mechanism in RRAM device with $\mathrm{Pt} / \mathrm{CoSiO}_{X} / \mathrm{TiN}$ structure," IEEE Electron Device Lett., vol. 32, no. 4, pp. 545-547, Apr. 2011.

[15] S. Kanga, S. Honga, C. R. Choeb, et al., "Preparation and characterization of epoxy composites filled with functionalized nanosilica particles obtained via sol-gel process," Polymer, vol. 42, no. 3, pp. 879-887, Feb. 2001. 